Prepared for the U.S. Department of Homeland Security

Domestic Nuclear Detection Office

under U.S. Department of Energy Contract DE-AC05-76RL01830

\title{
PVT Degradation Studies: Acoustic Diagnostics
}

Revision 0

Gerges Dib

Brian Tucker

Richard Kouzes

Phil Smith

April 2017

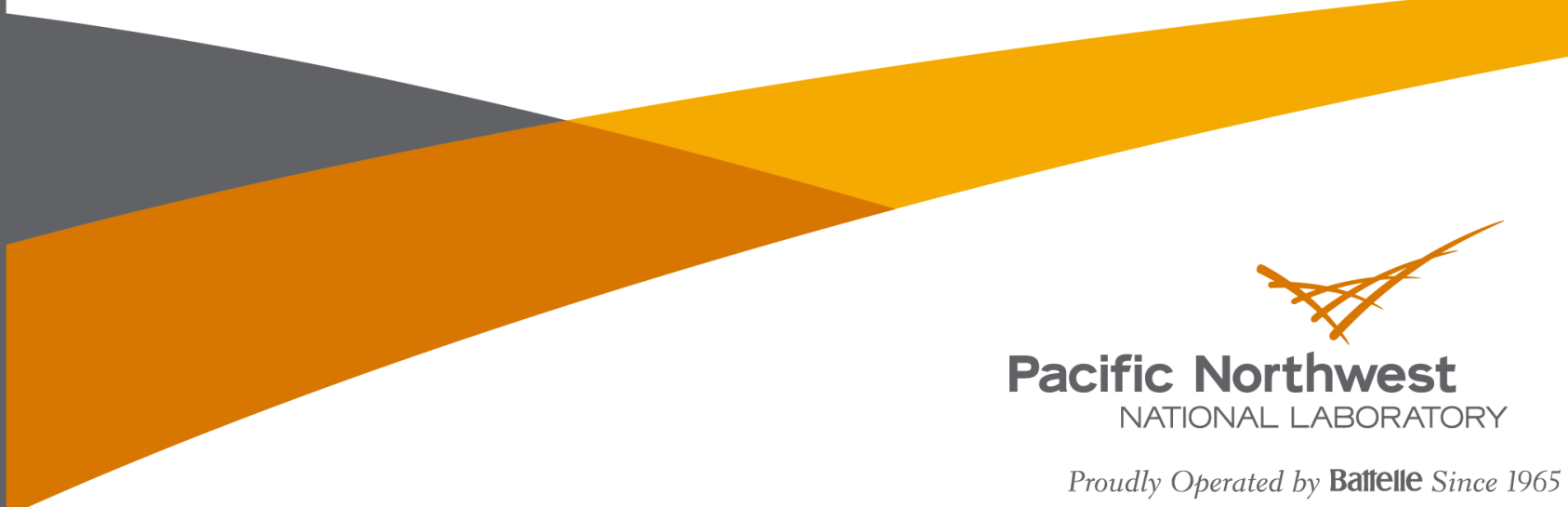




\title{
DISCLAIMER
}

This report was prepared as an account of work sponsored by an agency of the United States Government. Neither the United States Government nor any agency thereof, nor Battelle Memorial Institute, nor any of their employees, makes any warranty, express or implied, or assumes any legal liability or responsibility for the accuracy, completeness, or usefulness of any information, apparatus, product, or process disclosed, or represents that its use would not infringe privately owned rights. Reference herein to any specific commercial product, process, or service by trade name, trademark, manufacturer, or otherwise does not necessarily constitute or imply its endorsement, recommendation, or favoring by the United States Government or any agency thereof, or Battelle Memorial Institute. The views and opinions of authors expressed herein do not necessarily state or reflect those of the United States Government or any agency thereof.

\author{
PACIFIC NORTHWEST NATIONAL LABORATORY \\ operated by \\ BATTELLE \\ for the \\ UNITED STATES DEPARTMENT OF ENERGY \\ under Contract DE-AC05-76RL01830
}

Printed in the United States of America
Available to DOE and DOE contractors from the Office of Scientific and Technical Information,
P.O. Box 62, Oak Ridge, TN 37831-0062;
ph: (865) 576-8401
fax: $(865)$ 576-5728
email: reports@adonis.osti.gov

\begin{abstract}
Available to the public from the National Technical Information Service, U.S. Department of Commerce, 5285 Port Royal Rd., Springfield, VA 22161 ph: (800) 553-6847 fax: $(703) 605-6900$ email: orders@ntis.fedworld.gov online ordering: http://www.ntis.gov/ordering.htm
\end{abstract}

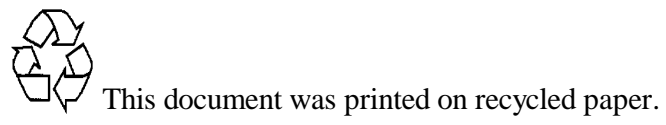




\title{
PVT Degradation Studies: Acoustic Diagnostics
}

\author{
Gerges Dib \\ Brian Tucker \\ Richard Kouzes \\ Phil Smith
}

April 2017

Prepared for

the U.S. Department of Homeland Security

Domestic Nuclear Detection Office

under U.S. Department of Energy Contract DE-AC05-76RL01830

Pacific Northwest National Laboratory

Richland, Washington 99352 


\section{Executive Summary}

Under certain environmental conditions, polyvinyl toluene (PVT) plastic scintillator has been observed to undergo internal fogging. This document reports on a study of acoustic techniques to determine whether they can provide a diagnostic for the fogging of PVT.

Different ultrasound techniques were tested for detecting the level of internal fogging in PVT, including wave velocity measurements, attenuation, nonlinear acoustics, and acoustic microscopy. The results indicate that there are linear relations between the wave velocity and wave attenuation with the level of internal fogging. The effects of fogging on ultrasound wave attenuation is further verified by acoustic microscopy imaging, where regions with fog in the specimen demonstrate higher levels of attenuation compared to clear regions. Results from the nonlinear ultrasound measurements were inconclusive due to high sensitivities to transducer coupling and fixture variabilities.

The results have shown a correlation (with a linear trend) between the wave speed and attenuation and fogginess level. In addition, acoustic microscopy obtained high-resolution images of the specimen surface. We used acoustic imaging to distinguish fogged and clear regions within the image. The imaging results further confirmed the attenuation measurements findings, where the attenuation level was higher in the fogged regions. The nonlinear ultrasound measurements were inconclusive since no clear trends were observed. This is mainly because nonlinear ultrasound measurements require absolute amplitude measurements, which are highly affected by small variations in transducer coupling and even fixture settings.

In summary, acoustic diagnostics are not likely to be an easily fieldable technology for determination of fogging of PVT-based gamma ray detectors, though permanently mounted sensors on the PVT could potentially monitor the development of fogging. 


\section{Contents}

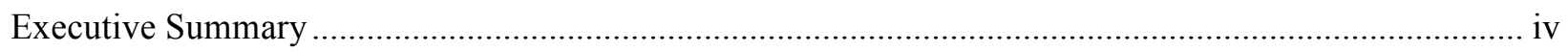

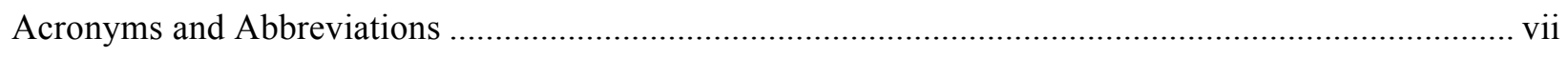

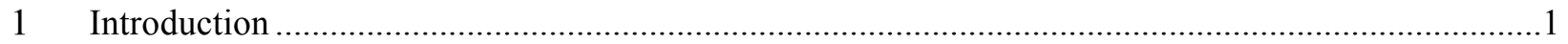

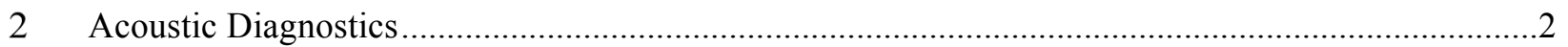

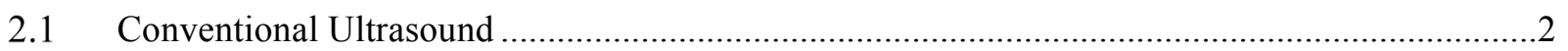

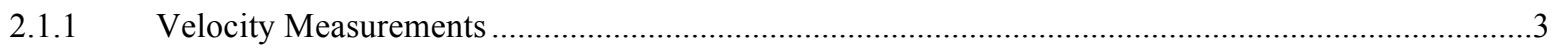

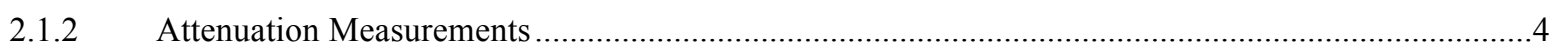

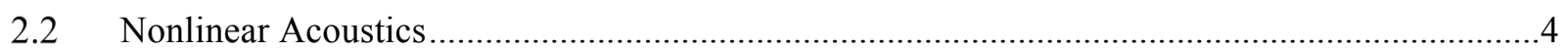

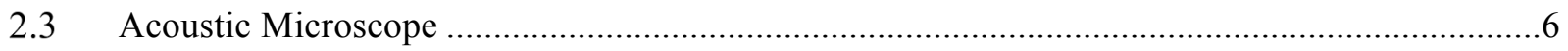

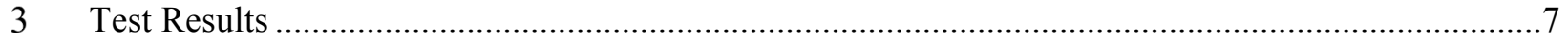

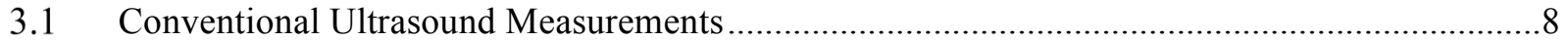

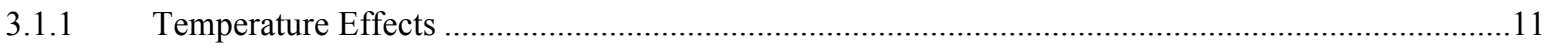

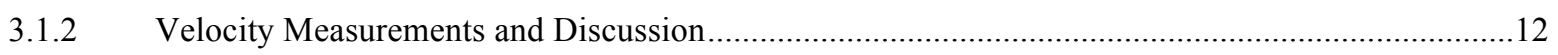

3.1.3 Attenuation Measurements and Discussion.................................................................................

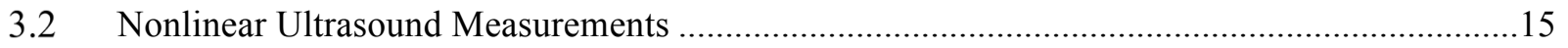

3.3 Acoustic Microscope Measurements .................................................................................. 17

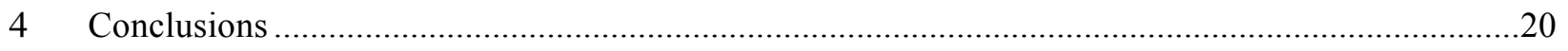

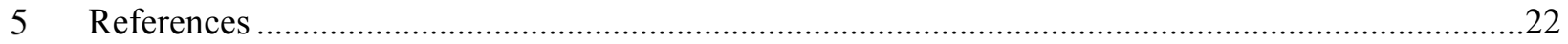

\section{Figures}

Figure 2.1. Typical experiment using conventional ultrasound for measuring wave speed and attenuation.

Figure 2.2. A typical signal received in normal beam pulse-echo mode measurements............................3

Figure 2.3. A through-transmission setup for measuring the nonlinearity parameter. ...............................5

Figure 2.4. Left: Example of a backscatter echo in the time domain. Right: Magnitude of the Fourier transform showing the spectrum for the fundamental and second harmonic signals........................6

Figure 3.1. The level of fogginess in the specimen at different times after removal from the cold chamber.

Figure 3.2. Top and side view of the specimen used for acoustic microscopy........................................

Figure 3.3. Experiment setup for the normal beam pulse-echo conventional ultrasound measurements......8

Figure 3.4. Transmitted square pulse by the pulser in conventional ultrasound experiments.....................9 
Figure 3.5. Measurements points for the coventional ultrasound experiments, looking at the top surface view of the specimen

Figure 3.6. Conventional ultrasound measurements on all dates.......................................................... 10

Figure 3.7. Effects of temperature on the received ultrasound echoes from the specimen back-wall.........11

Figure 3.8. Effects of temperature on velocity and attenuation within the pristine PVT specimen............11

Figure 3.9. Measurements from five different experiments, where the wave propagated through the length

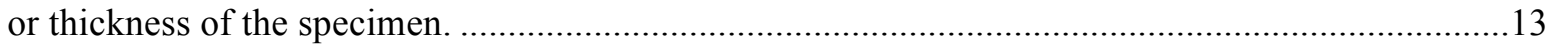

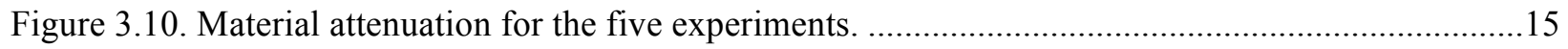

Figure 3.11. Experiment setup for the nonlinear ultrasound measurements...........................................16

Figure 3.12. Excitation signal for the nonlinear ultrasound experiments. ..............................................17

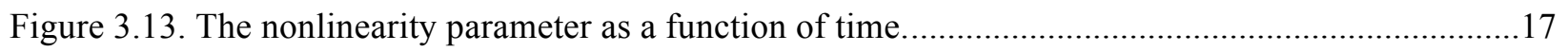

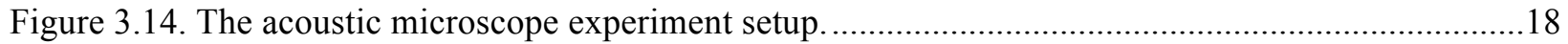

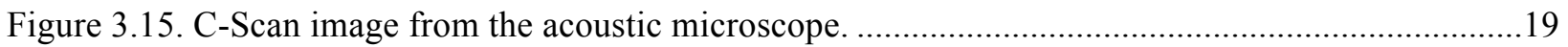

Figure 3.16. Averaging the acoustic microscope C-scan image along the $y$-axis (left) and the $\mathrm{x}$-axis

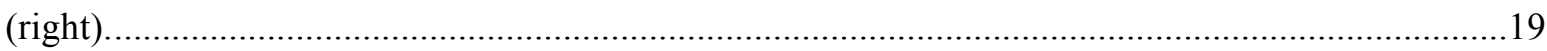

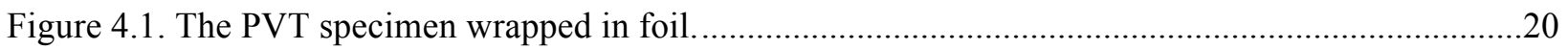




\section{Acronyms and Abbreviations}

$\begin{array}{ll}\text { AAT } & \text { Accelerated Aging Test } \\ \text { NLUT } & \text { nonlinear ultrasonic waves } \\ \text { PNNL } & \text { Pacific Northwest National Laboratory } \\ \text { PVT } & \text { polyvinyl toluene (plastic scintillator) } \\ \text { UT } & \text { ultrasound testing }\end{array}$

Page vii of vii 


\section{Introduction}

Polyvinyl toluene (PVT)-based gamma-ray detectors are used for a variety of applications. Some PVT panels, exposed to certain environmental conditions, have shown a reduction in performance due to absorption of moisture over years of deployment. The degradation manifests itself as internal fogging of the PVT (Cameron 2015).

Techniques to diagnose the degree of fogging in PVT, and its physical origin, are being investigated. Acoustic techniques are one approach being investigated to evaluate the condition of PVT.

This report discusses acoustic measurements on PVT samples that have been thermally treated for 60-90 days. We employed several different acoustic techniques including: velocity measurements, attenuation measurements, nonlinearity parameter measurements, and acoustic microscopy. Section 2 provides a brief description of each of the techniques used in this study, and Section 3 provides descriptions of the experimental measurements and the results. Section 4 gives conclusions and recommendations for future work based on the findings in this report. 


\section{Acoustic Diagnostics}

Ultrasound testing (UT) is a non-destructive testing technique that is based on the propagation of ultrasound waves in materials. Typical applications of UT use piezoelectric probes for transmitting short ultrasound pulses and a wave propagates through the material (Auld 1973; Hellier 2003; Rose 2004). When the wave is received, it holds information about the material due to its interaction with it, and with any flaws that might occur within it. Acoustic wave propagation in solids is a function of the mechanical properties of the solid and is affected by the macro and microstructure. Traditionally, changes in ultrasonic velocity and attenuation have been correlated with microstructural changes from various forms of degradation. Also, nonlinear elastic wave responses can provide improved sensitivity to accumulated damage. The development of internal fogging in PVT results in microstructural changes in the materials relative to ultrasound waves. This is because the sizes of voids that get filled with water (fog) are of the same size or smaller than the wavelength. This report investigates the sensitivity of different ultrasonic techniques to the level of internal fogginess in the specimen. The following first describes velocity and attenuation measurement techniques under the conventional ultrasound methods. Then, nonlinear ultrasound methods are described for detecting microstructural changes. A technique called acoustic microscopy is also described, which provides high-resolution imaging of specimens.

\subsection{Conventional Ultrasound}

A typical ultrasound inspection for measuring wave velocity and attenuation is shown in Figure 2.1. A transducer is directly coupled to the specimen using an ultrasound coupling gel. The transducer operates in duplex mode (also known as pulse-echo mode), where it both transmits and receives ultrasonic waves. The transducer transmits a short pulse into the specimen, which is reflected from the specimen's backwall to return to the transducer. A typical recorded signal using this measurement technique is shown in Figure 2.2. In this case, four echoes are received from the specimen's back wall with a sufficient signalto-noise ratio. If more than one echo is received from the specimen's back-wall, as is the case in Figure 2.2 , the wave velocity and attenuation can be computed by measuring the time difference and amplitude differences between consecutive echoes in the signal. The following subsections describe the methods used for computing wave velocity and attenuation.

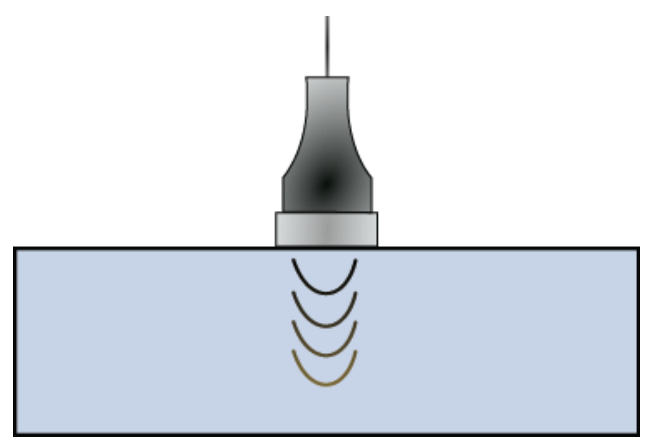

Figure 2.1. Typical experiment using conventional ultrasound for measuring wave speed and attenuation. 


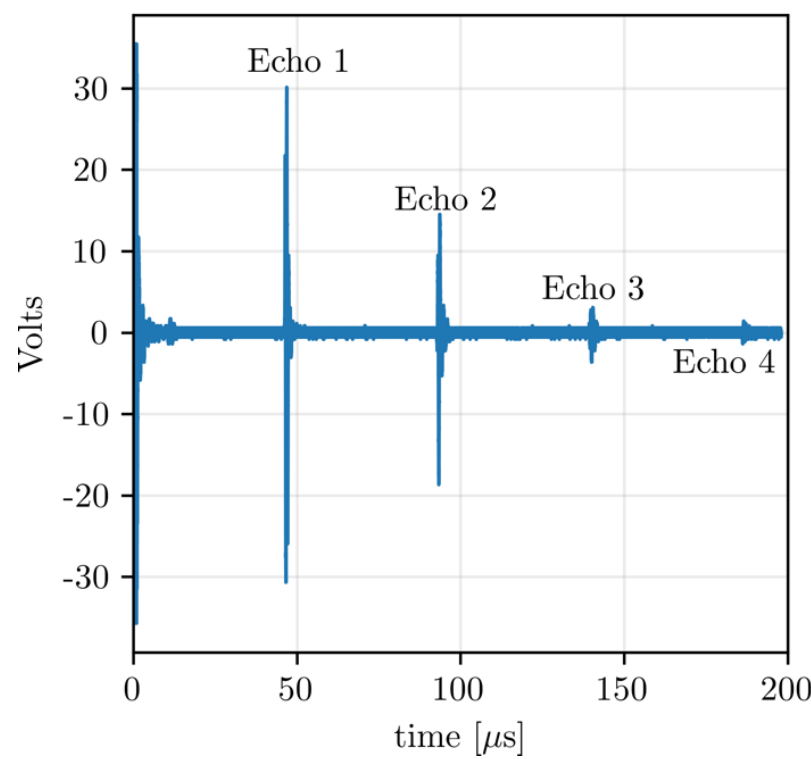

Figure 2.2. A typical signal received in normal beam pulse-echo mode measurements. The echoes are from the specimen's back wall .

\subsubsection{Velocity Measurements}

For a longitudinal elastic wave propagating in an isotropic solid medium, the wave speed is given by (Rose 2004):

$$
c_{L}=\sqrt{\frac{\lambda+2 \mu}{\rho}}
$$

Where $\lambda$ and $\mu$ are Lamè constants, which define the elastic mechanical properties of an isotropic material, and $\rho$ is the material density. The longitudinal wave velocity is solely a function of the material's mechanical properties. By measuring changes in the ultrasonic wave velocity, we can detect changes in the material's mechanical properties or its density. Thus, this method will detect any effects that fogginess in the PVT has on its macroscopic mechanical properties.

To compute the wave velocity, the wave time of flight and the propagation distance of the wave must be known. In the experiments, the propagation distance is known. The time of flight is computed by maximizing the cross-correlation between two consecutive echoes in the specimen (i.e., in Figure 2.2, the cross-correlation of echo 2 and echo 1 , echo 3 and echo 2 , etc....). If $s_{1}[n]$ is the digitized samples of echo 1 and $s_{2}[n]$ the digitized samples of echo 2 , then the time of flight is computed as:

$$
\arg \max _{k} \sum_{n=1}^{N} s_{1}[n] s_{2}[n+k] \quad k \in
$$

Where $N$ is the total number of samples in the signals, and $k$ is the number of sample shifts required to maximize the correlation between the two signals. The time of flight in seconds is then computed by multiplying $k$ with the data acquisition sampling time. The cross-correlation method provides a highly accurate measure of the time of flight, with accuracy on the order of the sampling time, which is $10^{-9} \mathrm{~s}$ $(1 \mathrm{~ns})$ for the data measurements collected in this report. 


\subsubsection{Attenuation Measurements}

When a wave propagates through a material, its intensity decreases with distance due to wave spreading and due to scattering and energy absorption by the material's microstructural inhomogeneity. The goal of attenuation measurements is to compute the coefficient of attenuation representing the viscoelastic effects due to material inhomogeneity. If we consider a circular piston source transducer operating in pulse-echo mode (see Figure 2.1), then the particle displacement of a longitudinal wave with normal incidence within an isotropic material can be expressed as (Schemerr and Song 2007):

$$
p(D, \omega)=\left\{1-\exp \left(j \frac{\omega a^{2}}{D c}\right)\left[J_{0}\left(\frac{\omega a^{2}}{D c}\right)-j J_{1}\left(\frac{\omega a^{2}}{D c}\right)\right]\right\} \exp (-\alpha D)
$$

Where $D$ is the propagation distance of the wave, $\omega$ is the circular frequency, $a$ is the transducer diameter, $c$ is the wave velocity, and $\alpha$ is the attenuation coefficient which we are interested in. $J_{0}()$ and $J_{1}()$ are Bessel's function of first kind of $0^{\text {th }}$ and $1^{\text {st }}$ order, respectively. This propagation model considers both the diffraction effects (signal spreading), and material viscoelastic effects. This model represents the wave propagation in the frequency domain (since it is a function of $\omega$ ). To compute the attenuation coefficient $\alpha$, the Fourier transform of each of the echoes in the signal is found and a nonlinear least squares fit to the amplitudes of the echoes to equation (0.1) at a given frequency $\omega$ is computed. If there are two consecutive echoes, then the first echo has propagation distance $\mathrm{D}$ and second echo has propagation distance 2D. Taking the ratios of the amplitudes of the two echoes and then their logarithm, and rearranging:

$$
\alpha=\frac{1}{D}\left[\frac{p(D, \omega)}{p(2 D, \omega)}-\ln \frac{1-\exp \left(j \frac{\omega a^{2}}{D c}\right)\left[J_{0}\left(\frac{\omega a^{2}}{D c}\right)-j J_{1}\left(\frac{\omega a^{2}}{D c}\right)\right]}{1-\exp \left(j \frac{\omega a^{2}}{2 D c}\right)\left[J_{0}\left(\frac{\omega a^{2}}{2 D c}\right)-j J_{1}\left(\frac{\omega a^{2}}{2 D c}\right)\right]}\right]
$$

where the values of $p(D, \omega)$ and $p(2 D, \omega)$ correspond to the Fourier transform amplitudes of the first and second echoes, respectively. When the PVT material has internal fogging, it is anticipated that the fogging level will change the microstructural characteristics of the material, which in turn will affect the attenuation coefficient of the material.

\subsection{Nonlinear Acoustics}

Nonlinear acoustics methods rely on the generation of harmonics from a monochromatic input. Nonlinear ultrasonic waves (NLUT) are sensitive to microscopic changes. This gives NLUT the potential to be used as an inspection technique in an early warning system. Generally, NLUT measurements use harmonic generation for evaluating the nonlinearity parameter of the material. A pitch-catch configuration is used, where a transmitter excites a high-amplitude wave through the structure at a given frequency $\omega_{0}$. Then, a receiver detects the wave propagating in the structure which now contains harmonic frequency components $\omega_{0}, 2 \omega_{0}$, and so on. Assuming a longitudinal wave propagating in the material, the nonlinear wave equation may be solved to show (de Lima and Hamilton 2003):

$$
\beta=\frac{8}{k^{2} x} \frac{A_{2}}{A_{1}^{2}}
$$


where $A_{2}$ is the amplitude of the second harmonic, $A_{1}$ is the amplitude of the fundamental frequency component, $k$ is the wavenumber, $x$ represents the propagation distance, and $\beta$ is the nonlinearity parameter. For a fixed frequency and propagation distance, measuring the amplitudes of the second harmonic and fundamental harmonic provides information about the nonlinearity parameter. The nonlinearity parameter has been shown to depend on the material microstructural characteristics (Cantrell 2009; Cantrell and Yost 2001). NLUT has been applied to the characterization of a range of damage mechanisms, including fatigue, thermal fatigue, and irradiation embrittlement (Cantrell and Yost 2001; Jhang 2000; Matlack et al. 2012).

A schematic of a typical measurement setup for NLUT measurements is shown in Figure 2.3. Typically, measurement techniques excite a longitudinal wave propagating through the thickness of the material using a pitch-catch configuration. In this setup, a probe transmits acoustic energy through the specimen and a receiving probe is placed on the opposite side of the specimen (through-transmission mode).

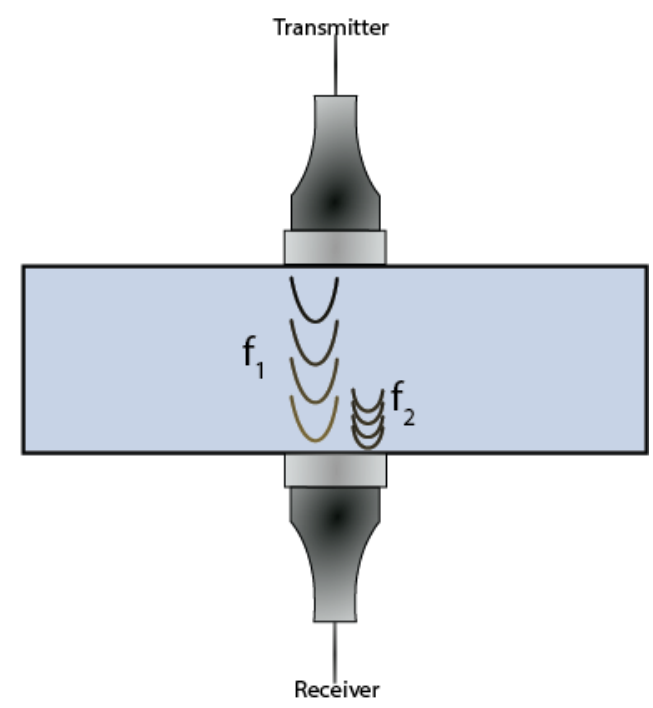

Figure 2.3. A through-transmission setup for measuring the nonlinearity parameter.

NLUT measurements most commonly utilize piezoelectric transducers for wave excitation and reception (Herrmann et al. 2006; Marino et al. 2016; Oruganti et al. 2007), since they are efficient in exciting highamplitude waves, relatively cheap and commercially readily available. Measurements using conventional piezoelectric transducers often do not provide the absolute value of the nonlinearity parameter because of transducer response function. Rather, piezoelectric transducers provide information on changes relative to a baseline. Consider a single echo received by the receiver in the experiment setup shown in Figure 2.3. An example of a received signal is shown in Figure 2.4. By computing the amplitude of the Fourier transform, we get the amplitudes of the fundamental $V_{1}$ and harmonic $V_{2}$ frequencies, such that:

$$
\frac{V_{2}}{V_{1}^{2}} \propto \beta
$$



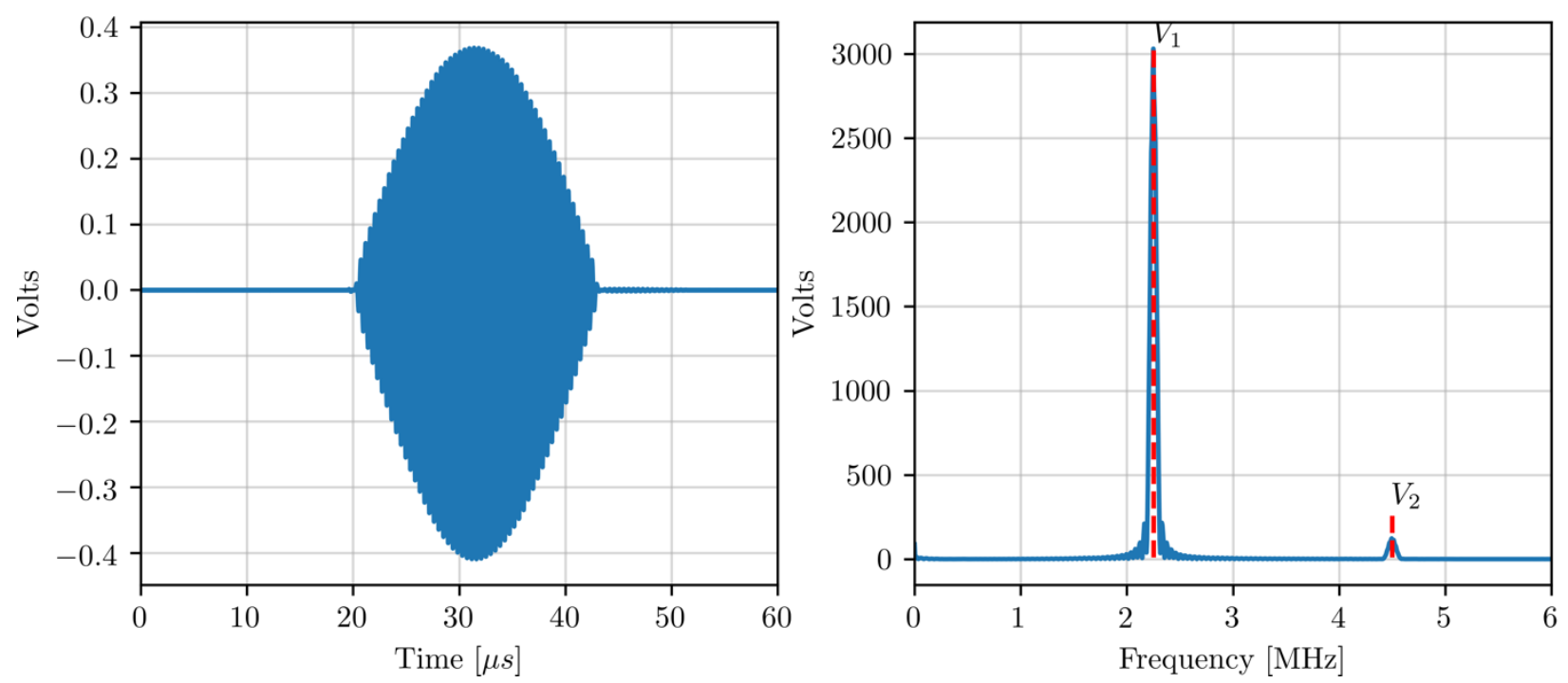

Figure 2.4. Left: Example of a backscatter echo in the time domain. Right: Magnitude of the Fourier transform showing the spectrum for the fundamental and second harmonic signals.

Amplitude measurements using conventional piezoelectric transducers depend on adequate coupling of the transducers. This poses challenges when making measurements on one or more specimens, as consistency of the coupling is difficult particularly in cases where the transducers are frequently removed and reapplied. As such, great care must be taken to obtain consistent results. Several authors have studied the amount of variability in such measurements and have proposed methods to minimize this variability (Liu et al. 2011; Sun et al. 2006).

\subsection{Acoustic Microscope}

The acoustic microscope is a device for conventional ultrasound measurements that produce highresolution images of the specimen using high-frequency-focused ultrasound transducers. Frequency ranges of transducers used in acoustic microscopy range from $1 \mathrm{MHz}$ up to $150 \mathrm{MHz}$. The acoustic microscope requires the specimen to be immersed in water for highly consistent coupling of the transducer with the specimen. A scanner is then used to scan the surface of the specimen. In this work, the acoustic microscope was used to image the surface of the PVT specimen in order to determine the areas of the specimen that still have fog in them, and the areas that do not. This type of inspection is different than the methods previously described. Using conventional and nonlinear ultrasound, the effect of specimen fogginess on ultrasound waves is measured by conducting measurements at different times, where the fog diffuses from the specimen with time. Using the acoustic microscope method, a specimen is imaged at only a single time to determine the fogginess level at that time. 


\section{Test Results}

The following describes the experiment setup used for each of ultrasound technique, and the corresponding results. To do the testing, $203.2 \times 203.2 \times 56 \mathrm{~mm}(8 \times 8 \times 2.2$ inch $)$ PVT specimen blocks were used. For the conventional and nonlinear ultrasound, the specimen (Eljen 9496-01-04) was kept hot and humid for 30 days followed by a cold cycle, repeated three times for a total of 90 days, as prescribed by the Accelerated Aging Test (AAT) method (Kouzes 2016). After the specimen was removed from the environmental chamber, ultrasound measurements were made every day for a week, and subsequently every week for a month. This pattern of measurement periods was made because the water content of the PVT decreases rapidly soon after the specimen is removed from the chamber. The water diffusion rate from PVT slows down with time after specimen are removed from the chamber. As an example, Figure 3.1 shows a specimen used for ultrasound measurements at different times after it had been out of the environmental chamber. A picture of a pristine specimen not subjected to the AAT is shown for comparison.
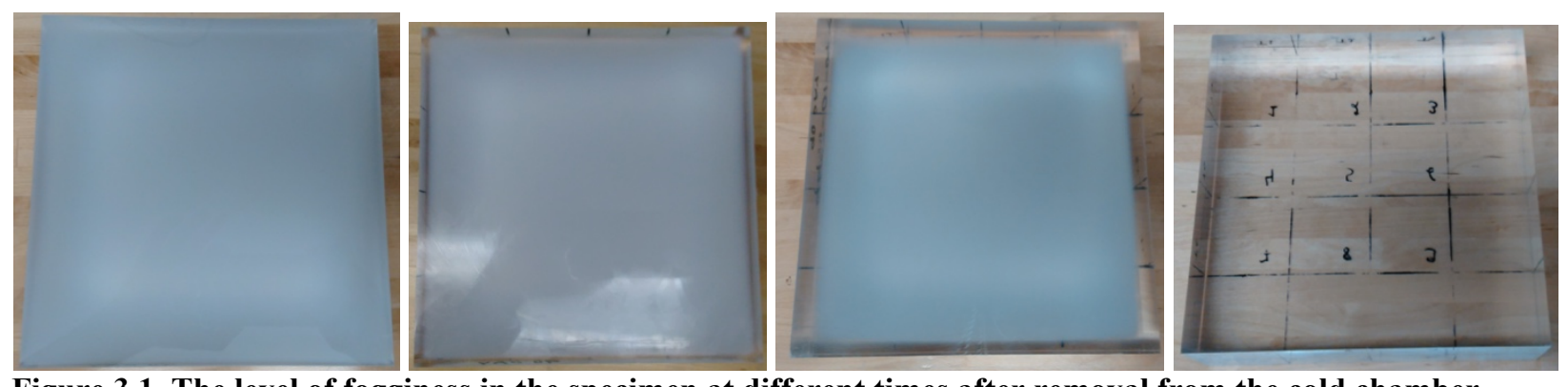

Figure 3.1. The level of fogginess in the specimen at different times after removal from the cold chamber. From left to right: Day 1, Day 3, Day 13, and a pristine sample not hot/cold treated (right).

As for acoustic microscopy, the specimen used for the measurements (Eljen 9496-01-08) was kept hot and humid for 60 days, with two cold cycles, per the AAT. The acoustic microscope measurements on the specimen were made after the specimen had been out of the cold chamber for 49 days. A picture of the specimen used is shown in Figure 3.2. The purpose of the acoustic microscope measurements was to detect the edge between regions where the specimen is clear of fog, and the region where it is foggy, as seen in Figure 3.2.
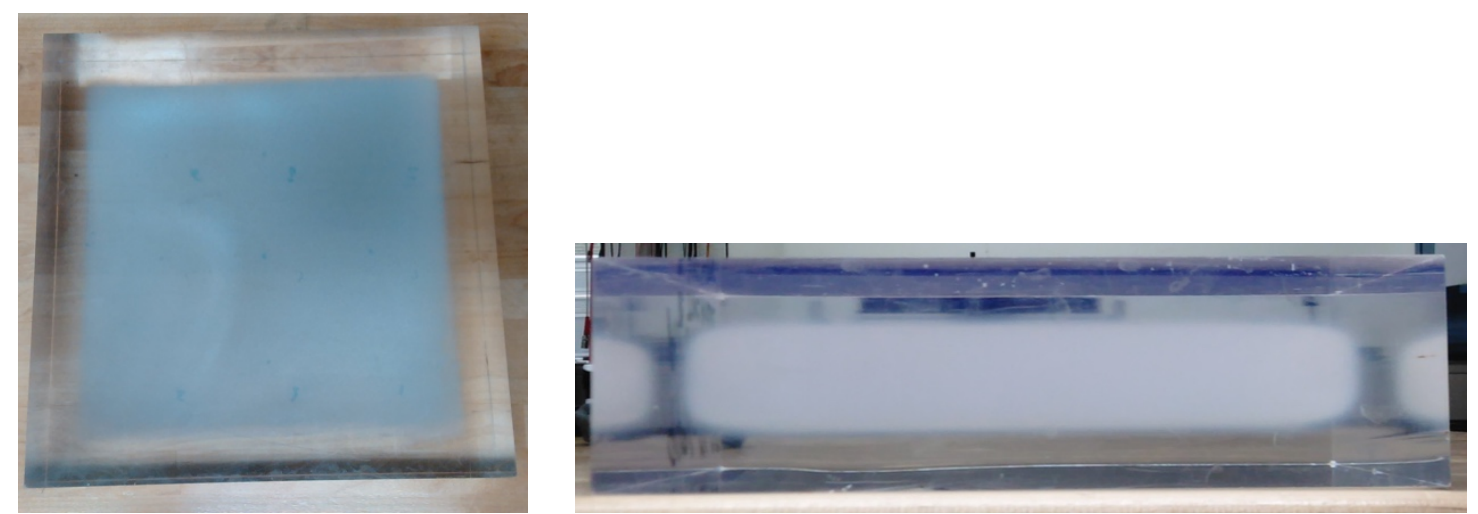

Figure 3.2. Top and side view of the specimen used for acoustic microscopy. This specimen had been out of the cold chamber for 49 days. 
In the following, the experiment setup that was used to obtain the different measurements, and the results obtained using the measurements are described, based on the concepts discussed in Section 2.

\subsection{Conventional Ultrasound Measurements}

A normal beam pulse-echo ultrasound measurement setup was used for the attenuation and velocity measurements. The experiment setup is shown in Figure 3.3. The Ritec SP-801A square pulser transmits a square pulse such as that shown in Figure 3.4 at a specified frequency to a piezoelectric transducer whose center frequency is the same as the excitation pulse. Since in these measurements we are operating in pulse-echo mode, the same transducer is used to receive the echoes which are reflected from the backwall of the specimen. A diplexer is used to route the received signal to an oscilloscope. To increase the signal-to-noise ratio, the average of 256 received signals is computed before recording the signal. The transducers were operated in normal beam mode, and thus they were directly coupled to the surface of the specimen using an ultrasound gel couplant.

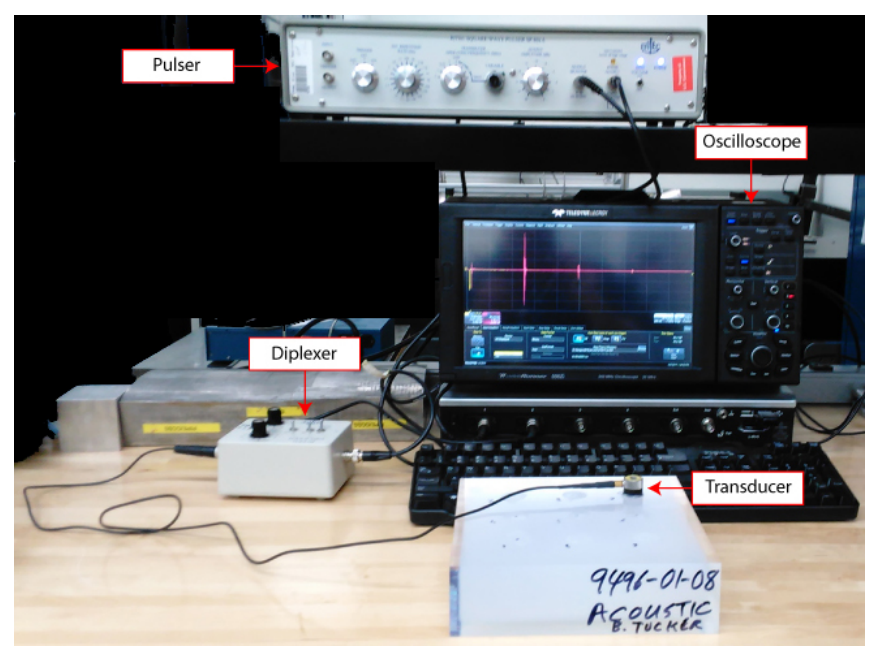

Figure 3.3. Experiment setup for the normal beam pulse-echo conventional ultrasound measurements. 


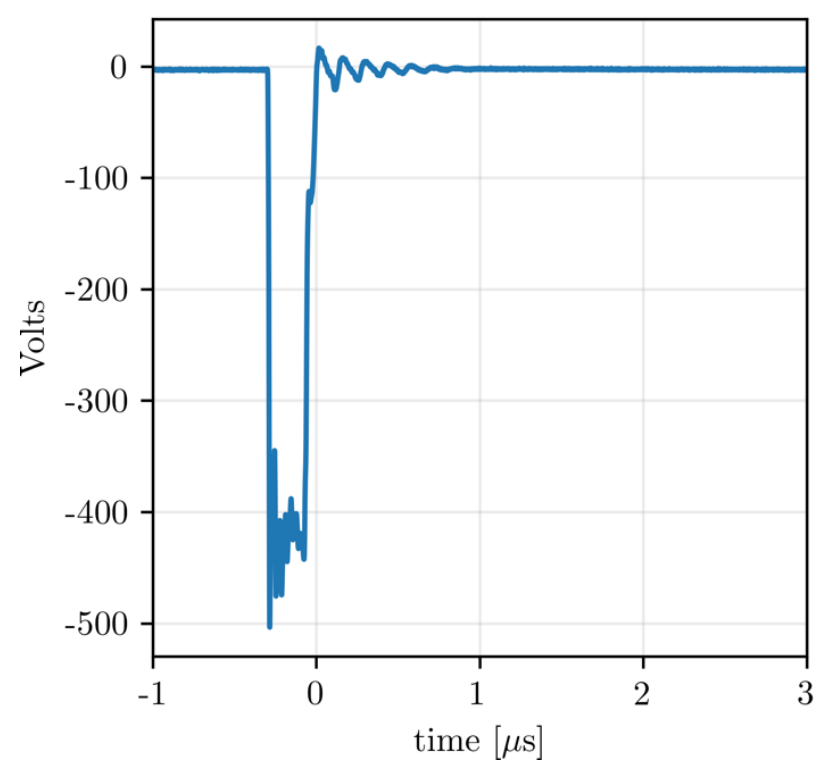

Figure 3.4. Transmitted square pulse by the pulser in conventional ultrasound experiments.

For each experiment, data was collected at a grid of nine different positions on the surface of the specimen as shown in Figure 3.5. We call those measurements through-thickness measurements, since the ultrasound wave propagates through the thickness of the specimen. They are labeled with numeric values from 1-9 in Figure 3.5. Three transducers with frequencies 2.25, 5, and $10 \mathrm{MHz}$ were used for the through-thickness measurements. All transducers had a 0.5 -inch diameter. In addition, through length pulse-echo measurements were made, where the transducer was positioned at the side of the specimen and the ultrasound wave propagated through the length of the specimen (203.2 $\mathrm{mm}$ or 8 inch). The measurement points for the through-length experiments are labeled T1-T6 in Figure 3.5. Two $12.7 \mathrm{~mm}$ (0.5 inch) diameter transducers with frequencies 1,2.25 MHz were used for the through-length measurements. The lower frequency transducer was used since it has less material attenuation, and thus we can obtain a signal with good signal-to-noise ratio after a $406.4 \mathrm{~mm}$ (16-inch) round trip through the specimen length.

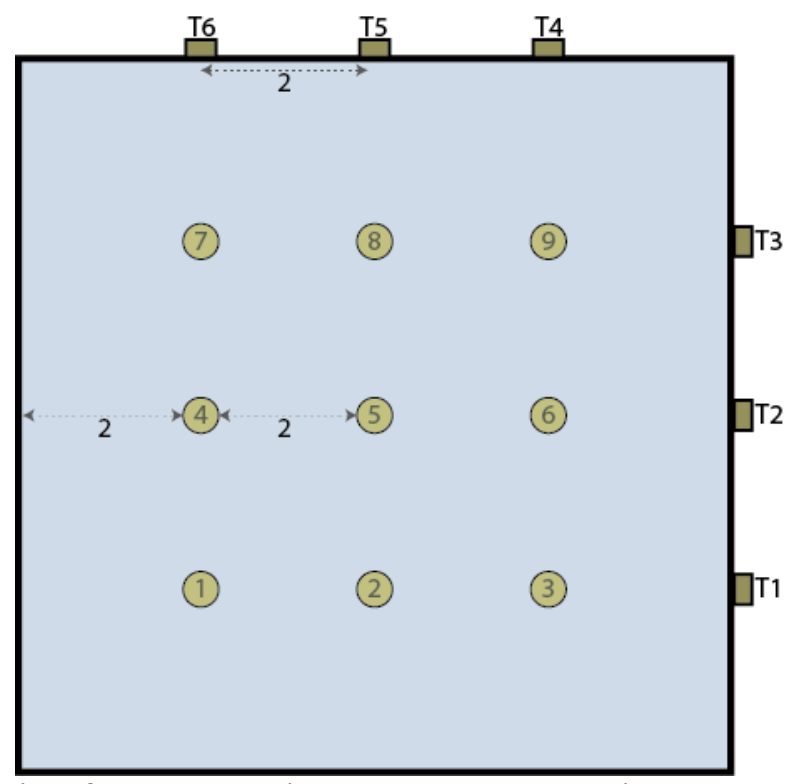

Figure 3.5. Measurements points for the coventional ultrasound experiments, looking at the top surface view of the specimen. The spacing between points is shown in inches. 
Before extracting the velocity and attenuation information from the measured signals, the raw signals obtained on different days were evaluated. The fogginess level in the specimen decreased as the days passed. Figure 3.6 shows the first and second back-wall echoes of the ultrasound measurements for through-thickness inspection using a $2.25 \mathrm{MHz}$ transducer, and at position 5 (see Figure 3.5). From Figure 3.6, it is readily observed that the first measurement obtained just after the specimen was taken out of the environmental chamber had a slightly different time of flight and attenuation than the consequent measurements. However, while doing the measurement right after removal from the chamber, the specimen temperature was still lower than room temperature, and it is speculated that this might be the dominant reason for changes in the signal, rather than its sensitivity to the fogginess level. To investigate the effect of temperature on velocity and attenuation in PVT, a study was made as described in the next section.

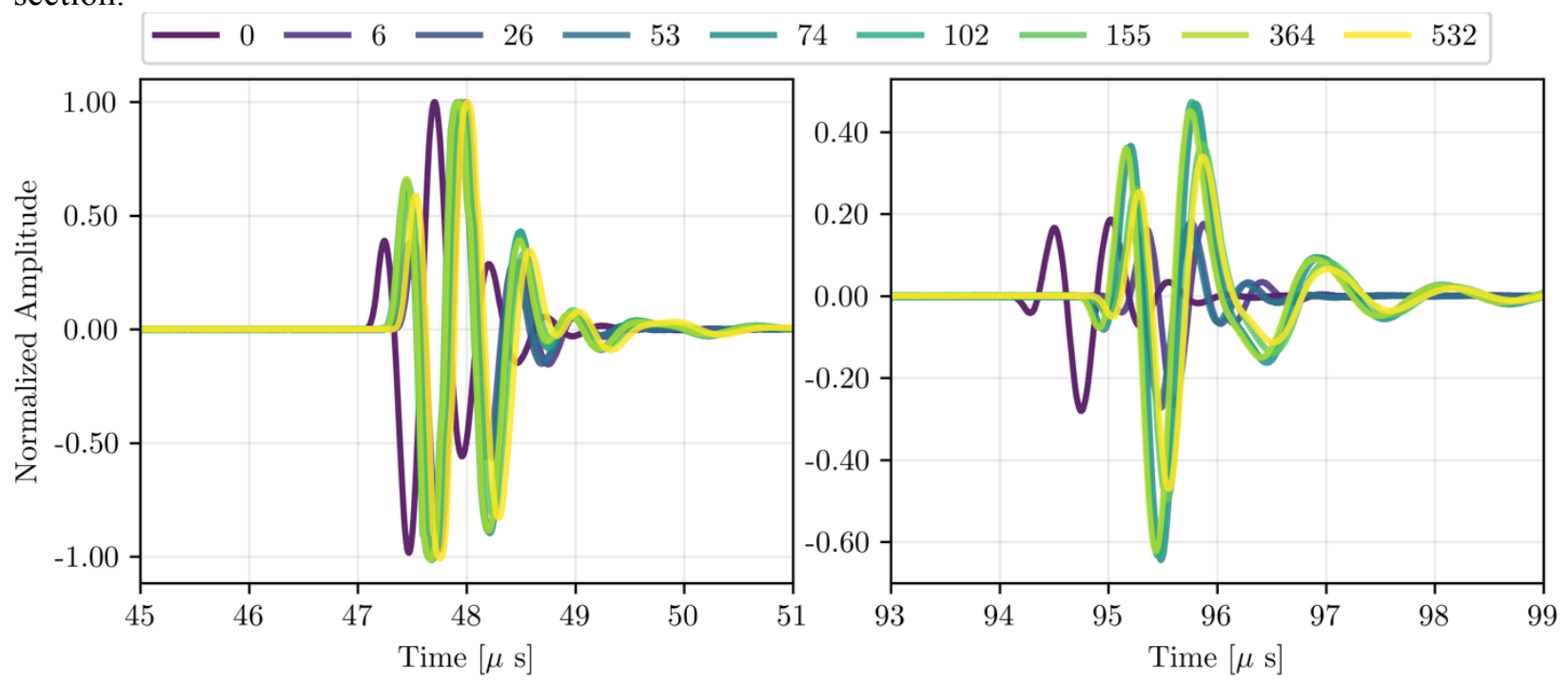

Figure 3.6. Conventional ultrasound measurements on all dates. The legend shows the time the measurement was recorded in hours after the specimen was taken out of the cold chamber. Left: The first echo from specimen back-wall, right: second back-wall echo. All the ultrasound signals were normalized by the maximum amplitude of the first echo, so all first echoes have an amplitude of 1. 
3.2 Temperature Effects A pristine PVT specimen (not hot/cold treated) was cooled down to $7^{\circ} \mathrm{C}$. Then, a $2.25 \mathrm{MHz}$ transducer was fixed on the specimen surface at position 5 (see Figure 3.5), and pulse-echo ultrasound signals were recorded every twenty seconds, as the specimen warmed back up to room temperature. The specimen temperature was recorded periodically using an infrared thermometer, so that the effect of temperature on the measured attenuation and wave speed could be tracked. The first and second echoes from the specimen back-wall are shown in Figure 3.7.
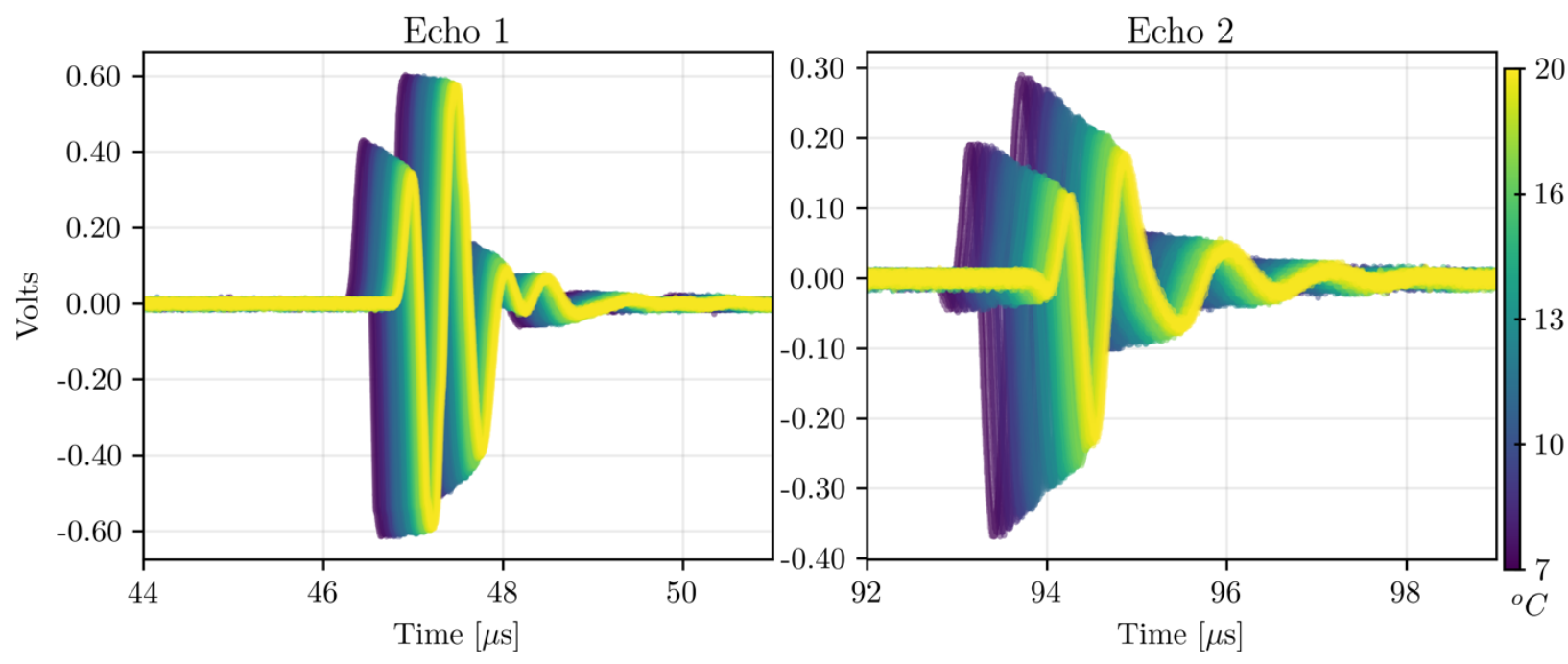

Figure 3.7. Effects of temperature on the received ultrasound echoes from the specimen back-wall.

The signals shown in Figure 3.7 were then processed using to the methods described in Section 2 to extract the wave velocity and attenuation, and the results are shown in Figure 3.8. The mean changed in wave velocity is approximately $1.3 \%$ for temperatures between $7^{\circ} \mathrm{C}$ and $20^{\circ} \mathrm{C}$. Although this change appears to be low, it is in fact significant in terms of material velocity variation. On the other hand, the material attenuation had a mean variation of $28.8 \%$. This significant variation in attenuation with temperature would affect the accuracy of the inspection measurements on the foggy specimen when it is first taken out of the cold chamber.
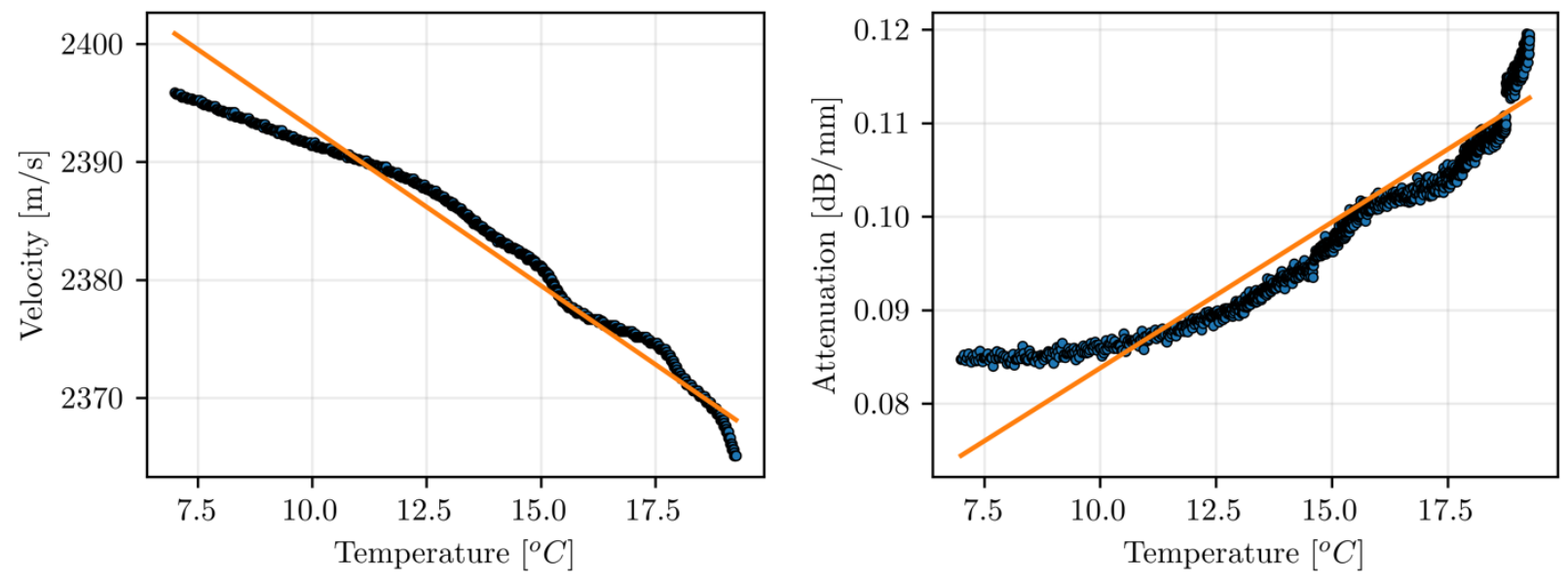

Figure 3.8. Effects of temperature on velocity and attenuation within the pristine PVT specimen. 


\subsubsection{Velocity Measurements and Discussion}

As we have discussed in Section 2, the time difference between the back-wall echoes was used to compute the wave velocity with high accuracy. If more than two back-wall echoes are present, the mean velocity is computed based on the time of flight between consecutive echoes. The computed wave velocities are shown Figure 3.9 for the five experiments considered: through-thickness measurements with three probes at different frequencies, and through-length measurements with two probes at different frequencies. To observe the relative change in velocity with increasing time (decreasing internal fogginess level), the velocity values were normalized as:

$$
c_{\text {normalized }}=\frac{c-\mu_{c}}{\sigma_{c}}
$$

Where $\mathrm{c}$ is the wave velocity, $\mu_{\mathrm{c}}$ is the mean wave velocity across all times, and $\sigma_{\mathrm{c}}$ is the standard deviation of all velocity across all times. At each time point, the uncertainty ranges are computed as the standard deviation of measurements at different positions on the plate (nine positions for throughthickness measurements and six positions for through-length measurements). This uncertainty is due to tolerances in the specimen thickness and length which is used to compute the wave velocity. The specimen thickness was measured with a $0.3 \mathrm{~mm}$ tolerance, explaining the uncertainties in velocity measurements at different positions on the specimen. Also, signal noise and distortion affects time of flight computation accuracy. The five experiments in Figure 3.9 show an increasing trend of velocity versus time in a logarithmic fashion. To that extent, the measurements were fitted using nonlinear least squares by a logarithmic function:

$$
c=p_{1} \ln p_{2} t+p_{3}
$$

Where $t$ is the time, and $p_{1}, p_{2}$ and $p_{3}$ are the parameters to be computed using nonlinear regression. The fog within the specimen also diffuses in a logarithmic fashion, where the water content within the specimen diffuses rapidly once the specimen is removed from the cold chamber, and then the rate decreases with time. Thus, our initial velocity measurement results give evidence that there is a measureable linear relation between wave speed and the fogginess level within the specimen. The wave velocity decreases linearly with increasing fogginess level. This trend was especially true for the experiment with the lowest frequency at $1 \mathrm{MHz}$ (top left in Figure 3.9).

Note that the first measurement acquired at time zero is outside the range shown in Figure 3.9, except for the $10 \mathrm{MHz}$ probe, where the initial measurement is visible. This is due to the effect of low temperature, since the specimen was still cold after removal from the environmental chamber. This effect was explained in Section 3.2.1 (see Figure 3.8). Once the specimen reached room temperature, it was predicted that the random variations between day to day measurements can be attributed to small changes in room temperature. However, since the laboratory room temperature randomly fluctuates near a mean value, those effects average themselves out in the long run, and do not affect the overall trend. 

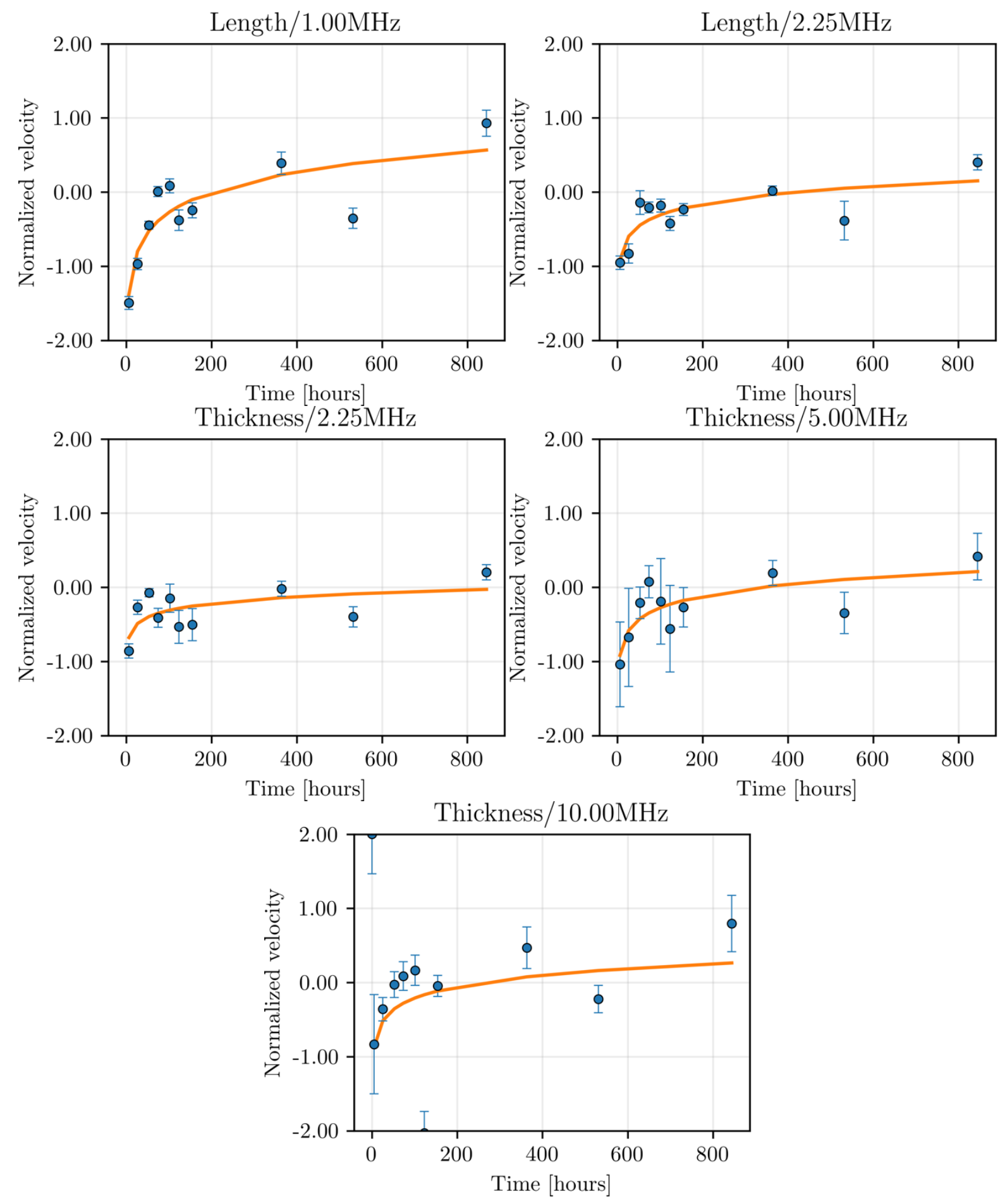

Figure 3.9. Measurements from five different experiments, where the wave propagated through the length or thickness of the specimen. 


\subsubsection{Attenuation Measurements and Discussion}

We also used the experiments to extract the material attenuation parameter using the methods we have discussed in Section 2. The normalized material attenuation is shown Figure 3.10 for the five experiments: through-thickness measurements with three probes at different frequencies, and throughlength measurements with two probes at different frequencies. The attenuation was normalized similar to the normalization described by equation $(0.3)$ for the velocity measurements. Also, a logarithmic regression line of the form given in equation (0.4) is shown in Figure 3.10. Similar to the plots for the velocity measurements, the uncertainty ranges at each time are computed as the standard deviation of measurements at different positions on the sample. This uncertainty in attenuation measurement is mostly due to variations in coupling conditions between the probe and specimen which affects signal amplitudes and distortion. All the five experiments show a logarithmic relation with time, especially for the $1 \mathrm{MHz}$ and $10 \mathrm{MHz}$ experiments, where the uncertainty level was lower. This correlation provides evidence that the attenuation level has a linear relation with the fogginess level, where attenuation increases within increasing fogginess. 

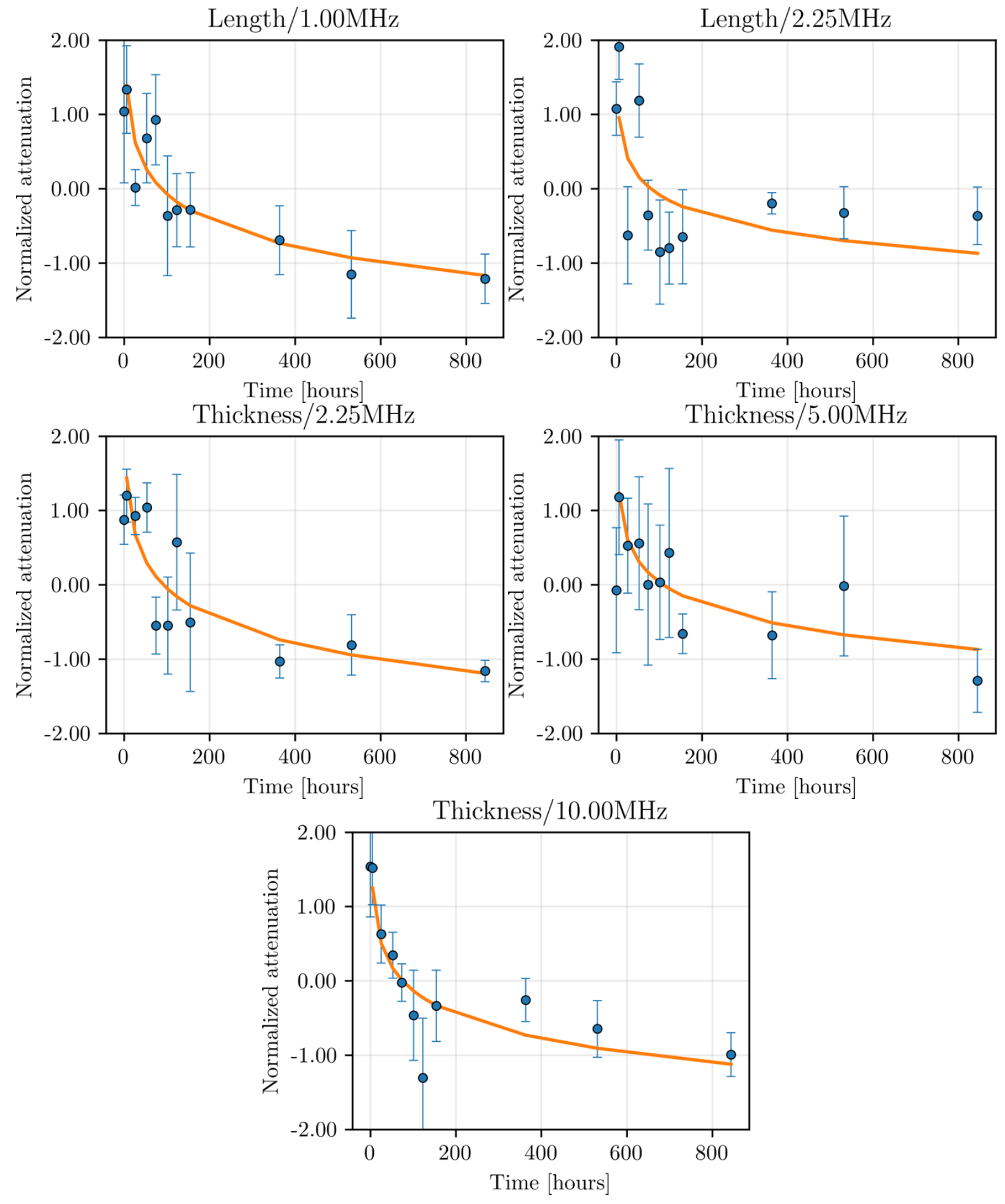

Figure 3.10. Material attenuation for the five experiments.

\subsection{Nonlinear Ultrasound Measurements}

The nonlinear ultrasound measurements used different equipment than the conventional ultrasound measurements. The difference in equipment allows sending pulses with higher voltage level, and also for 
generating band-limited excitations, rather than short square pulses. The experimental setup for the nonlinear measurements is shown in Figure 3.11. A Ritec Snap RAM-5000 gated power amplifier is used to generate a 50 cycle sine tone burst at a $2.25 \mathrm{MHz}$ center frequency as shown in Figure 3.12. The output of the power amplifier is connected to a $50 \mathrm{ohm}$ load to maximize the power transmitted to the transmitting transducer. A $2.25 \mathrm{MHz}$ frequency transducer is used as a transmitter. At the receiving side, a $5 \mathrm{MHz}$ transducer is used to maximize its sensitivity to the second harmonic generation. The receiving transducer is connected directly to an oscilloscope, where 256 signal averages are taken to maximize the signal-to-noise ratio. Since nonlinear ultrasound depends on the absolute amplitude of the signals, special care was taken to maximize the consistency of the measurements, and minimize the coupling effects on the signal amplitudes between different measurements. A fixture is used to align the two transducers at the two opposite surface of the specimen. At the transmitted side, the probe was connected to a load cell to measure the amount of force that the probe is exerting on the specimen surface. On the receiving side, the probe is connected to a spring loaded threaded knop. The knop on the receiver side was tightened until the load cell on the transmitter side indicated that the force was $8 \mathrm{lb}$.

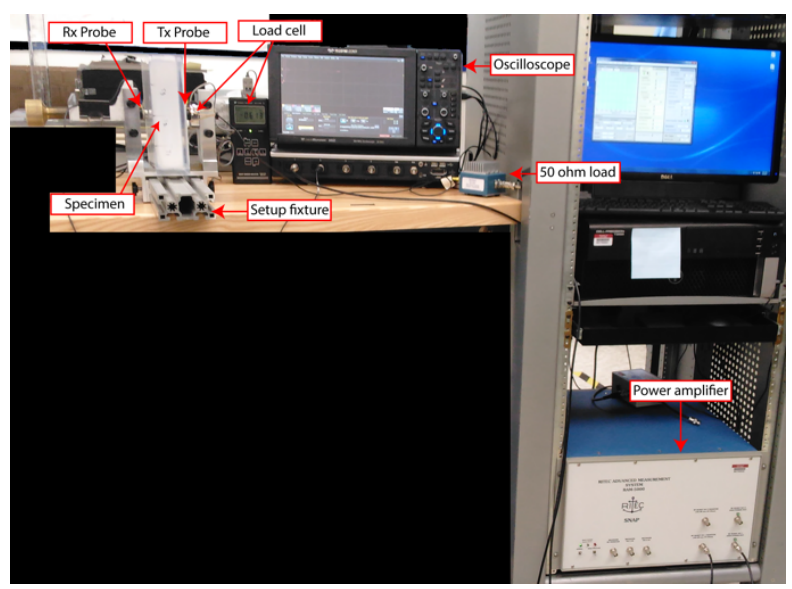

Figure 3.11. Experiment setup for the nonlinear ultrasound measurements.

The measurements were collected at position 5 (see Figure 3.5), which is the center of the specimen. Measurements were taken every day for seven days after the specimen was taken out of the environmental chamber, and then weekly for three weeks. Five different measurements were taken each day to quantify the variation in the measurements due to the transducers coupling conditions. For each measurement, the specimen was removed, and the coupling (ultrasound gel) was reapplied to the transducer, and the measurement procedure was repeated. 


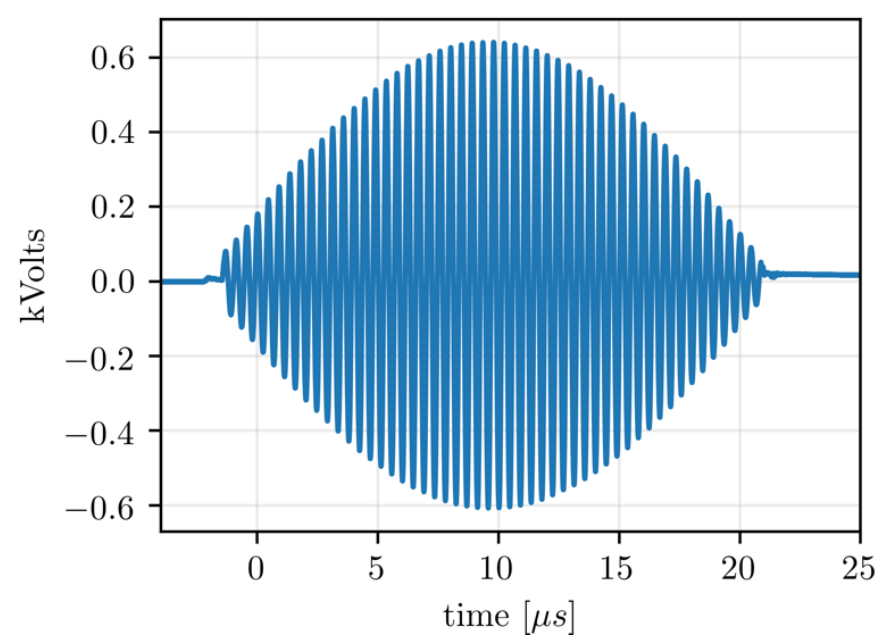

Figure 3.12. Excitation signal for the nonlinear ultrasound experiments.

The nonlinearity parameter was computed using the methods describes in Section 2 . The normalized nonlinearity parameter is shown in Figure 3.13. At each time, five measurements were made to capture the variability of the measurements due to transducer coupling variations. The linear regression line shows a relation that is proportional to the level of fogginess in the specimen. The nonlinearity parameter measurements show significant uncertainties and there appears to be no significant correlation between the nonlinearity parameter and fogginess level. Due to the large uncertainties and the possible biases that might occurs in the amplitude measurements, there is no clear indication if the change in the fit is due to real changes in the nonlinearity parameter or just experimental uncertainties and biases.

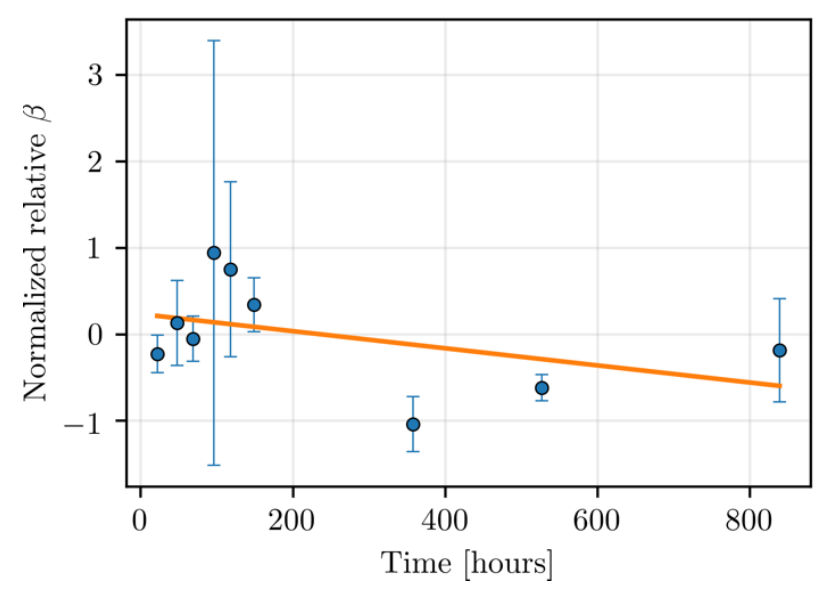

Figure 3.13. The nonlinearity parameter as a function of time. The dashed line show the linear regression of the data.

\subsection{Acoustic Microscope Measurements}

The acoustic microscope experiment setup is shown in Figure 3.14. The specimen is immersed in a water bath so that the transducer is water coupled to the specimen. The surface of the specimen is scanned to obtain a C-scan image (top view). The C-scan allows seeing the boundaries between the fogged and clear regions in the specimen. A quarter inch diameter, $20 \mathrm{MHz}$ probe with $25.4 \mathrm{~mm}$ focal length was used for the scanning. The high frequency of the probe allows greater resolution, with wavelength on the order of $120 \mu \mathrm{m}$. 

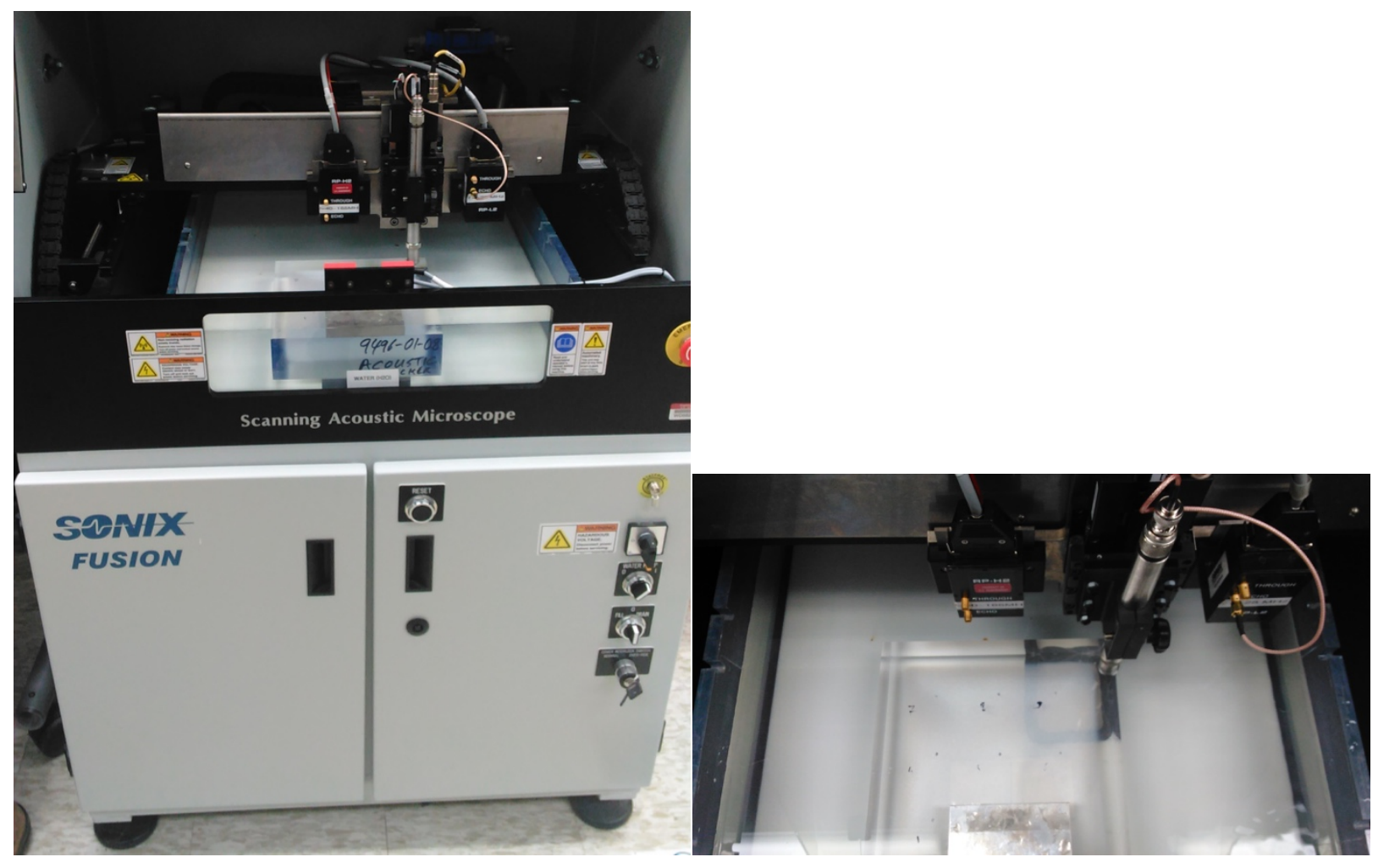

Figure 3.14. The acoustic microscope experiment setup.

The specimen was scanned in both direction with $0.1 \mathrm{~mm}$ steps. The C-scan image of the specimen is shown in Figure 3.15. The $\mathrm{C}$-scan image does have higher amplitudes near the edge of the specimen which is clear. This is the region outside the rectangular contour in Figure 3.15. To further see this change of amplitude at the fogged/clear edge, the image was averaged across each of its axes. The results of averaging the $\mathrm{C}$-scan produces a line scan as shown in Figure 3.16. At the $20 \mathrm{~mm}$ location, where the specimen transitions from being clear to foggy, the signal amplitude decreased. At the $180 \mathrm{~mm}$ location, the signal amplitude went back up since the specimen has another edge where it transitions from being foggy to clear. Those results further reinforce the finding from the attenuation measurements using conventional ultrasound, where a linear relation between attenuation level and the specimen fogginess was found. 


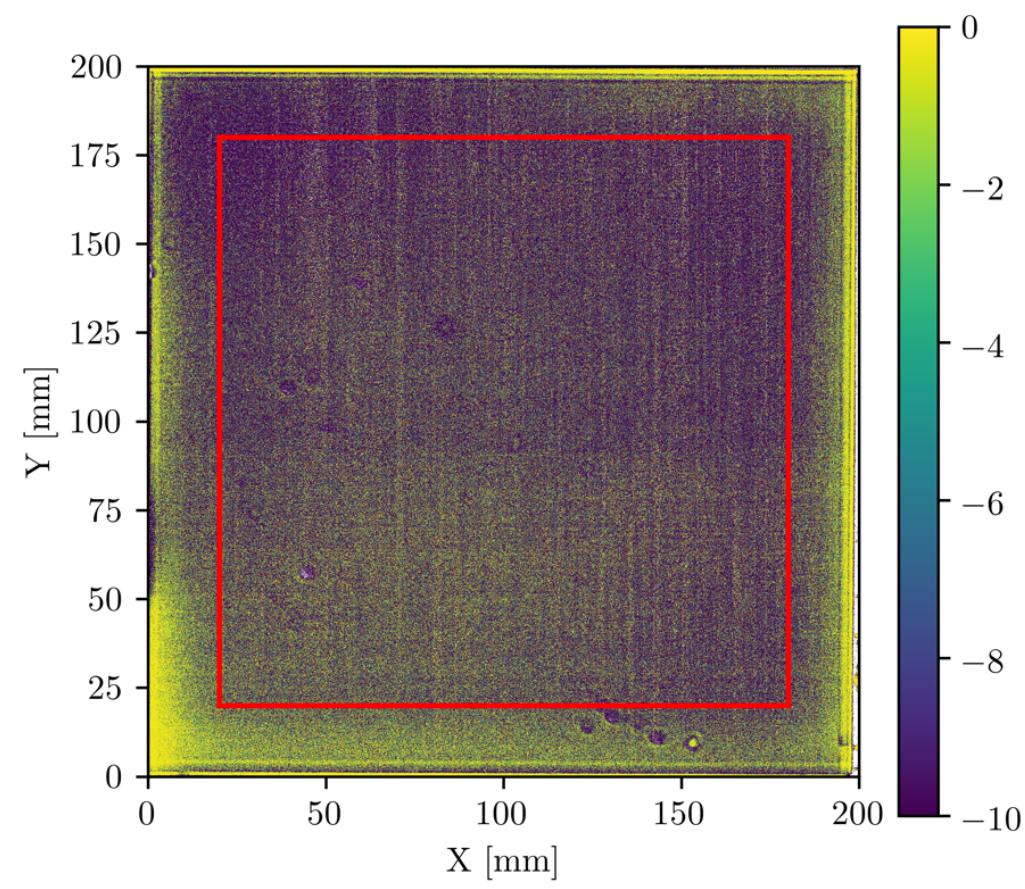

Figure 3.15. C-Scan image from the acoustic microscope. The red rectangle indicates the region separating the non-fogged region (outside) from the fogged region (inside). The color scale is in $\mathrm{dB}$.

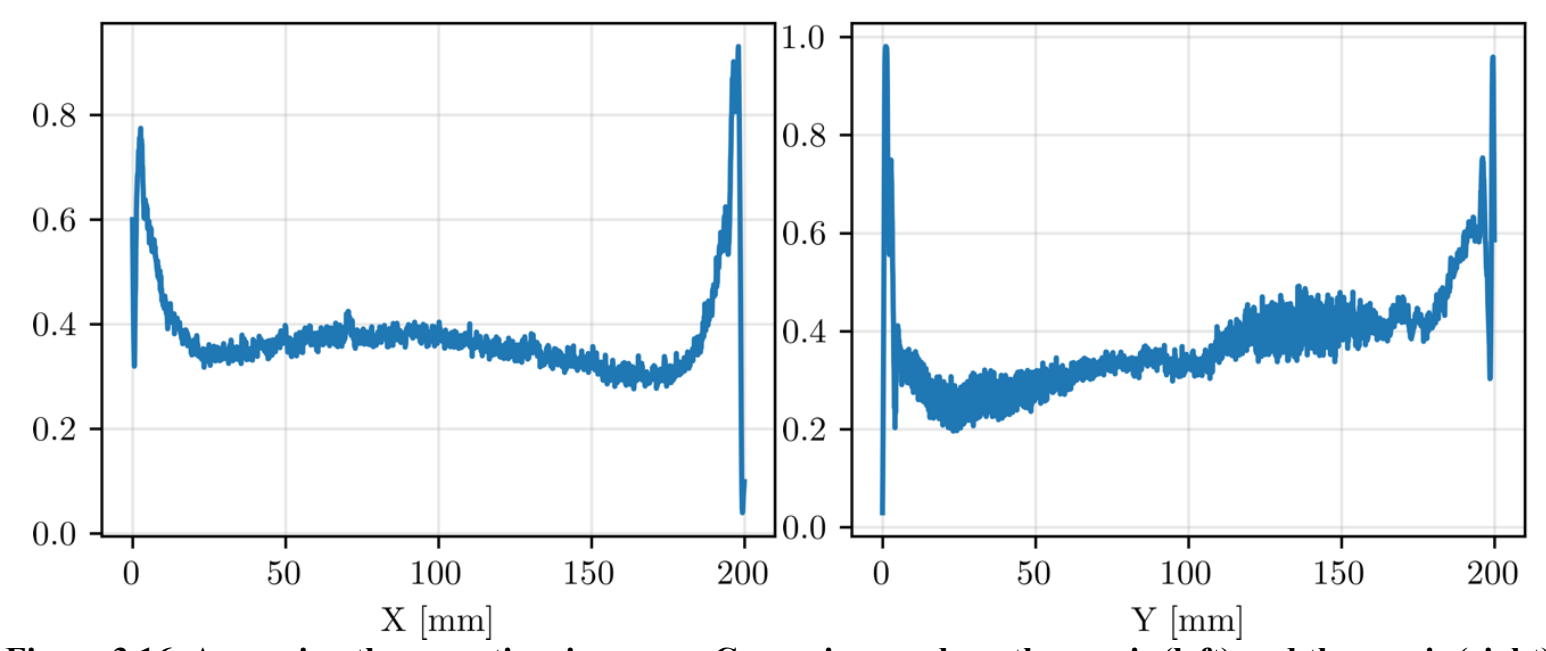

Figure 3.16. Averaging the acoustic microscope $C$-scan image along the $y$-axis (left) and the $x$-axis (right). 


\section{Conclusions}

In this report, linear, nonlinear, and high-resolution imaging ultrasound methods were used for detecting the level of fogginess in PVT. The results have shown a correlation (with a linear trend) between the wave speed and attenuation and fogginess level. In addition, acoustic microscopy obtained highresolution images of the specimen surface. Acoustic imaging was used to distinguish fogged and clear regions within the image. The imaging results further confirmed the attenuation measurements findings, where the attenuation level was higher in the fogged regions. The nonlinear ultrasound measurements were inconclusive since no clear trends were observed. This is mainly because nonlinear ultrasound measurements require absolute amplitude measurements, which are highly affected by small variations in transducer coupling and even fixture settings.

The initial measurements using conventional ultrasound techniques show that they are a possible technique for detecting PVT fogginess, but only if the measurement uncertainty is reduced. Moreover, further investigation is required to quantify the reliability of such techniques in terms of the minimum detectable fog levels and the confidence in detection, since only heavily fogged samples were used in this study. Since transducer coupling is a major source of uncertainty in ultrasound, one option would be to use transducers that are permanently bonded to the sample to be monitored. The bonding of transducers to the structure eliminates coupling variability. Moreover, such a technique would allow obtaining higher accuracy nonlinear measurements. However, since ultrasound signals are highly sensitive to environmental and operating conditions, it would still be required to develop techniques to compensate for those effects. In practice, a permanently mounted acoustic system provides the advantage of the possibility of continuous monitoring and automated quantification of fogging levels in PVT materials.

One of the advantages of using ultrasound is its ability to penetrate through thin coverings over the material. Thus, it may be possible to use ultrasound to inspect PVT wrapped in foil and tape, as would be the case for fielded PVT, without the need to remove such coatings if sufficient consistent pressure is applied. The preliminary investigations showed that it is possible to transmit and receive ultrasound signals from the PVT specimen investigated in Section 3, even if it is wrapped in a foil as shown in Figure 4.1. However, no tests were done to investigate the effects of tape with foil wrapping on the sensitivity of ultrasound to fogging levels, and this is left for future investigations.

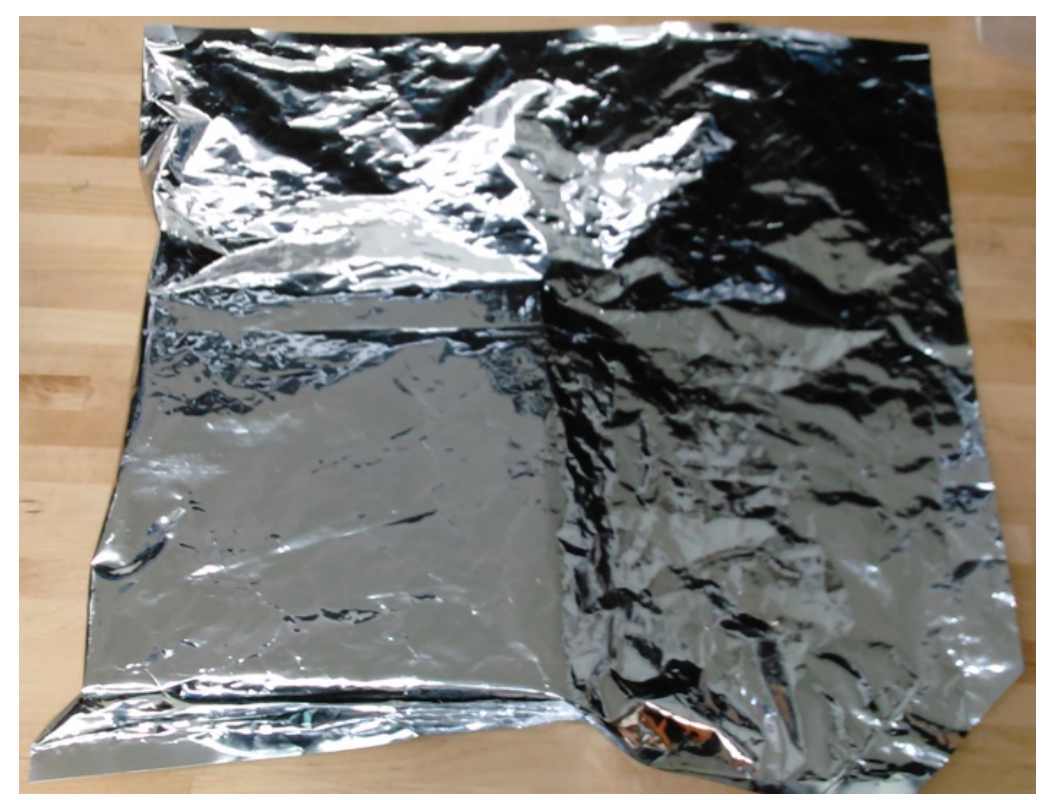

Figure 4.1. The PVT specimen wrapped in foil. 
In addition to the points mentioned above, future work could include two ultrasound-based methods that would possibly increase the sensitivity and applicability of acoustic techniques:

- In all the conventional ultrasound measurements conducted in this study, longitudinal wave transducers were used. Another possibility is to use shear wave transducers that would have higher sensitivity due to their smaller wavelength, which is nearly half that of the longitudinal waves. The smaller wavelength would be more affected by changes in the material attenuation.

- Conventional ultrasound methods use point measurements on the specimen, and thus it would be difficult to provide complete coverage for large structures. The use of surface waves, which travel along the surface of the specimen, could solve this problem and thus cover the surface area of the specimen rather than just a single point. Surface waves would be sensitive to changes near the surface of the specimen, up to a single wavelength depth. Lower frequencies can be used to provide deeper penetration across the thickness of the material. For example, the surface wave speed in PVT is approximately $1100 \mathrm{~m} / \mathrm{s}$, which corresponds to a $11 \mathrm{~mm}$ penetration depth at $100 \mathrm{kHz}$.

In summary, acoustic diagnostics are not likely to be an easily fieldable technology for determination of fogging of PVT-based gamma ray detectors, though permanently mounted sensors on the PVT could potentially monitor the development of fogging. A cost benefit analysis could determine the feasibility of this method. 


\section{References}

Auld, B A. 1973. Acoustic Fields and Waves in Solids. Volume I. John Wiley \& Sons.

Van der Auweraer, Herman, and Bart Peeters. 2003. "International Research Projects on Structural Health Monitoring: An Overview." Structural Health Monitoring 2(4): 341-58.

Cameron RJ, BG Fritz, C Hurlbut, RT Kouzes, A Ramey, and R Smola. 2014. "Fogging in polyvinyl toluene." IEEE Transactions on Nuclear Science, 62:1-4 (February 2015).

Cantrell, John H. 2009. "Nonlinear Dislocation Dynamics at Ultrasonic Frequencies." Journal of Applied Physics 105(4): 43520. http://scitation.aip.org/content/aip/journal/jap/105/4/10.1063/1.3081972.

Cantrell, John H., and William T. Yost. 2001. "Nonlinear Ultrasonic Characterization of Fatigue Microstructures." International Journal of Fatigue 23: 487-90. http://ac.elscdn.com/S0142112301001621/1-s2.0-S0142112301001621-main.pdf?_tid=a3e7101e-1793-11e5a4dd-00000aacb35d\&acdnat=1434836038_5f9fd97be96472124733160dbcecdd72.

Hellier, Charles J. 2003. Handbook of Nondestructive Evaluation. MCGraw-Hill.

Herrmann, Jan et al. 2006. "Assessment of Material Damage in a Nickel-Base Superalloy Using Nonlinear Rayleigh Surface Waves.” Journal of Applied Physics 99(12): 124913. http://scitation.aip.org/content/aip/journal/jap/99/12/10.1063/1.2204807.

Jhang, Kyung-young. 2000. "Applications of Nonlinear Ultrasonics to the NDE of Material Degradation." IEEE Transactions on Ultrasonics, Ferroelectrics and Frequency Control 47(3): 540-48.

Kouzes, Richard. 2016 Accelerated Aging Test for Plastic Scintillator Gamma Ray Detectors. PNNL25719, Pacific Northwest National Laboratory, Richland, Washington.

de Lima, W.J.N., and M.F. Hamilton. 2003. "Finite-Amplitude Waves in Isotropic Elastic Plates." Journal of Sound and Vibration 265(4): 819-39. http://linkinghub.elsevier.com/retrieve/pii/S0022460X02012609 (October 13, 2014).

Liu, Siming, Anthony J Croxford, Simon a Neild, and Zhenggan Zhou. 2011. "Effects of Experimental Variables on the Nonlinear Harmonic Generation Technique." IEEE transactions on ultrasonics, ferroelectrics, and frequency control 58(7): 1442-51. http://www.ncbi.nlm.nih.gov/pubmed/21768028.

Marino, Daniel et al. 2016. "Using Nonlinear Ultrasound to Track Microstructural Changes due to Thermal Aging in Modified 9\%Cr Ferritic Martensitic Steel.” NDT and E International 79: 46-52. http://dx.doi.org/10.1016/j.ndteint.2015.12.002.

Matlack, K. H. et al. 2012. "Evaluation of Radiation Damage Using Nonlinear Ultrasound.” Journal of Applied Physics 111(5): 109-12.

Oruganti, Ramkumar K. et al. 2007. "Quantification of Fatigue Damage Accumulation Using Non-Linear Ultrasound Measurements." International Journal of Fatigue 29(9-11): 2032-39. http://ac.elscdn.com/S0142112307000412/1-s2.0-S0142112307000412-main.pdf?_tid=4eebd0ce-ffe5-11e4a448-00000aab0f01\&acdnat=1432232286_5841906f226b6c412bc8c13037ebc26c.

Rose, Joseph L. 2004. Ultrasonic Waves in Solid Media. Cambridge University Press.

Schemerr Jr., Lester W, and Sung-jin Song. 2007. Ultrasonic Nondestructive Evaluation Systems: Models and Measurements. Springer.

Sun, L., S. S. Kulkarni, J. D. Achenbach, and S. Krishnaswamy. 2006. "Technique to Minimize CouplantEffect in Acoustic Nonlinearity Measurements." Journal of Acoustical Society of America 120(5). http://scitation.aip.org/docserver/fulltext/asa/journal/jasa/120/5/1.2354023.pdf?expires=1432678791 $\& \mathrm{id}=\mathrm{id} \&$ accname $=2097007 \&$ checksum $=851$ ACFC54A88F3113E9956DBAD1D30B8. 


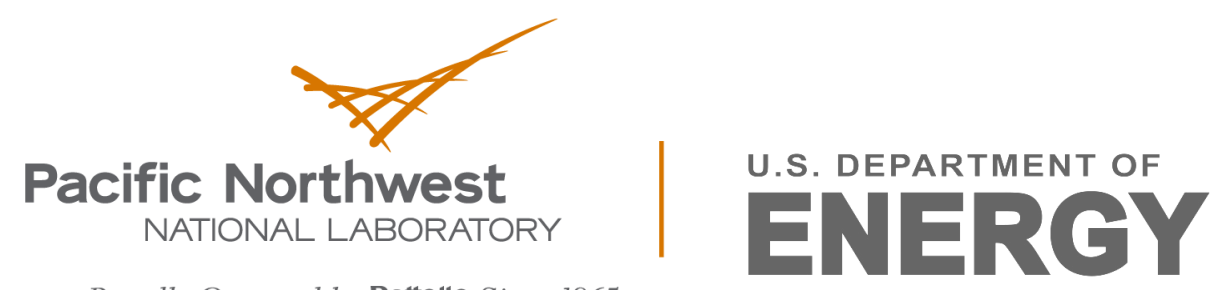

Proudly Operated by Battelle Since 1965

902 Battelle Boulevard

P.O. Box 999

Richland, WA 99352

1-888-375-PNNL (7665)

www.pnnl.gov 